\title{
Reflets
}

Revue ontaroise d'intervention sociale et communautaire

\section{Mauvais traitements infligés aux ainés}

\section{Rachel Ouellette}

Volume 2, numéro 1, printemps 1996

Contrer la violence subie par les femmes et les enfants en milieu familial

URI : https://id.erudit.org/iderudit/026119ar

DOI : https://doi.org/10.7202/026119ar

Aller au sommaire du numéro

Éditeur(s)

Reflets : Revue ontaroise d'intervention sociale et communautaire

ISSN

1203-4576 (imprimé)

1712-8498 (numérique)

Découvrir la revue

Citer cet article

Ouellette, R. (1996). Mauvais traitements infligés aux ainés. Reflets, 2(1),

184-187. https://doi.org/10.7202/026119ar

Tous droits réservés (C) Reflets : Revue ontaroise d'intervention sociale et communautaire, 1996

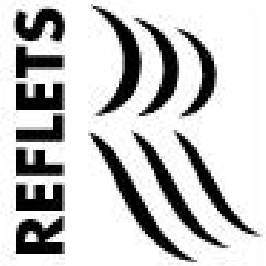

Ce document est protégé par la loi sur le droit d'auteur. L’utilisation des services d'Érudit (y compris la reproduction) est assujettie à sa politique d'utilisation que vous pouvez consulter en ligne.

https://apropos.erudit.org/fr/usagers/politique-dutilisation/ 


\section{Mauvais traitements infligés aux aînés}

\section{Rachel $\mathbf{O}$ uellette}

D irectrice de programme pour les services en français, $C$ onseil sur le vieillissement d' 0 ttawa-C aleton

\section{Le projet}

Les mauvais traitements infligés aux personnes âgées ne sont pas un phénomène nouveau. Son ampleur au $\mathrm{C}$ anada a fait l'objet de plusieurs enquêtes, dont celle de Podnieks et al., (1990), qui révèle qu'au moins $4 \%$ des aînés de notre pays sont victimes de mauvais traitements. U ne enquête ontarienne, réal isée auprès de 678 organismes, révèle que 61 \% d'entre eux, ont dépisté des cas confirmés ou présumés de mauvais traitements au cours des six mois précédant l'étude (ministère des Affaires civiques, 1991). $R$ écemment, une enquête menée par le C onseil sur le vieillissement (1995), auprès d'intervenants d'un large éventail d'organismes, révélait qu'un nombre total de 93 cas présumés de mauvais traitements avaient été identifiés sur une période de 12 semaines. Les données de cette dernière enquête sont présentées dans un document intitulé R ésultats de l'inventaire des cas de violence envers les personnes âgées.

Les mauvais traitementsinfligés aux personnes âgées prennent différentes formes; il peut s'agir d'agression physique ou psychologique, d'exploitation financière, de non-respect des droits individuels ou de négligence. II semble que l'exploitation financière soit la forme la plus courante. $Q$ uelle qu'en soit la forme, il 
demeure que les mauvais traitements infligés aux aînés représentent un problème important ayant des répercussions sur les services de santé, les services sociaux et l'aide juridique.

Depuis 1986, cette problématique est l'une des priorités du Conseil sur le vieillissement d'O ttawa-C arleton. D ans le but d'examiner ce problème social complexe, le Conseil a d'abord mis sur pied un groupe de travail. En 1993, avec l'appui de la Fondation Trillium de l'O ntario, le C onseil sur le vieillissement a entrepris un projet d'une durée de deux ans, visant à développer des outils et des structures qui permettent de répondre aux besoins communautaires reliés au problème des mauvais traitements infligés aux aînés.

\section{Le manuel}

Pour pouvoir identifier les cas de mauvaistraitements et répondre aux besoins qu'ils engendrent, il est essentiel de se doter d'outils et de connaissances supplémentaires. Les intervenantsont d'ailleurs confirmé ce besoin. C'est dans cet esprit que le Conseil sur le vieillissement a développé un manuel intitulé L es mauvais traitements infligés aux aînés: interventions possibles et ressources disponibles. La conception de ce manuel a été rendue possible grâce à la précieuse collaboration de nombreux bénévoles comprenant des aînés, des aidants naturels, des professionnels offrant des services aux aînés, et des personnes intéressées à la question du vieillissement. L'objectif principal est de sensibiliser davantage à la problématique des mauvais traitements infligés aux aînés, et d'offrir des stratégies destinées à aider les personnes qui travaillent auprès des personnes âgées, et qui peuvent un jour être confrontées à cette situation difficile.

Les prestateurs de services peuvent jouer un rôle clé dans la prévention des mauvais traitements infligés aux personnes âgées, ainsi que dans l'élimination des facteurs contribuant aux situations abusives. Le guide propose donc des stratégies préventives 
d'intervention, et présente les facteurs de risque qui prédisposent les familles à infliger les mauvais traitements.

Pour les intervenants, on insiste également sur l'importance, de maintenir une attitude professionnelle, et on propose desfaçons de faire face à ses réactions personnelles. U ne section du manuel traite également du profil des aînés à risque élevé, et des indices qui peuvent aider à identifier les mauvais traitements.

Enfin, on présente des directives générales sur les modalités d'intervention tant auprès de la victime que de l'agresseur. 0 n indique également, quels peuvent être les obstacles à l'intervention, comment développer des protocoles; on fournit en annexe des outils d'évaluation, des études de cas, des plans d'intervention et une liste des ressources disponibles pour intervenir dans les cas de mauvais traitements à l'égard des ainés. Ce précieux document est en vente au C onseil sur le vieillissement.

$D$ 'autres réalisations ont également été accomplies au cours des deux années de travail du Conseil sur le vieillissement sur cette question. Parmi celles-ci, mentionnonsl'établissement d'un bureau de conférenciers, qui a offert ses services à environ 350 personnes de la communauté, le développement d'un abri d'urgence mettant 6 lits à la disposition des personnes âgées victimes de mauvais traitements au Service de santé des Soeurs de la $C$ harité, une campagne d'information pour sensibiliser le public à l'existence du D istress $C$ entre et de Tel-aide $O$ utaouais (ces organismes ont des lignes téléphoniques pour répondre aux appels despersonnes âgées en situation de crise), le développement de deux dépliants d'information sur les mauvais traitements infligés aux aînés et sur les endroits où trouver de l'aide et, le développement d'un outil permettant de dépister et de traiter les cas de mauvais traitements (ce travail est toujours en cours). 


\section{Conclusion}

Les mauvais traitements obligés des personnes âgées sont une préoccupation importante sur laquelle il est essentiel de se pencher dès maintenant. II s'agit d'un phénomène qui a été maintenu trop longtemps dans le silence, la négation et l'isolement. C'est grâce à la prévention et à la concertation que l'on arrivera à faire face à ce problème et à trouver des solutions.

«a prévention des mauvais traitements et de la négligence envers les personnes âgées est un défi communautaire qui ne peut être résolu par le biais d'une seule personne ou d'une simple approche. Il faudra un effort communautaire pour créer un environnement qui réaffirme le droit des aînés à l'autodétermination, au respect et à la dignité.» (Lukawiekdi, 1992)

\section{Bibliographie}

CONSEIL SUR LE VIEILLISSEM ENT - OTTAWA-CAR LETON (1995). L es mauvais traitements infligés aux aînés: interventions possibles et ressources disponibles, $O$ ttawa, $C$ onseil sur le vieillissement d'O ttawa-Carleton.

CONSEIL SU R LE VIEILLISSEM EN T - OTTAWA-CAR LETON, (1995a). R ésultats de l'inventaire des cas de violence envers les personnes âgées, $O$ ttawa, $C$ onseil sur le vieillissement d'O ttawaCarleton.

LUKAW IECKI, T. (1993). C ommunity A wareness and R esponse: A buse and N eglect of 0 Ider A dults, Santé Canada, Division de la santé mentale.

M IN ISTÈR E DES AFFAIR ES CIVIQ UES (1991). A R eview of C ommunity/Program R esponses to E Ider $A$ buse in $O$ ntario, $O$ ttawa, $O$ ffice des personnes âgées.

PO D N IEKS, E. et K. Pillemer (1990). N ational Survey on A buse of the Elderly in C anada, Toronto, $R$ yerson Polytechnical Institute. 